\title{
Modelling of joint crowd-structure system using equivalent reduced-DOF system
}

\author{
Jackie Sim ${ }^{\mathrm{a}, *}$, Anthony Blakeborough ${ }^{\mathrm{b}}$ and Martin Williams ${ }^{\mathrm{b}}$ \\ ${ }^{\mathrm{a}}$ Mott MacDonald, St Anne House, Wellesley Road, Croydon CR9 2UL, UK \\ ${ }^{\mathrm{b}}$ Department of Engineering Science, University of Oxford, UK
}

\begin{abstract}
For human assembly structures in which the mass of the crowd is significant compared to that of the structure, it is necessary to model the passive crowd as a dynamic system added to the main structural system. Earlier work by the authors has analysed the frequency response of a joint crowd-structure system in which the structure is treated as a single degree-of-freedom (SDOF) system and the seated and standing crowds are each modelled as a two degree-of-freedom (2DOF) system. It was found that the occupied structure has dynamic properties different to the empty structure. This paper investigates representing the joint crowd-structure system as an equivalent reduced-DOF system that would have the advantage of simplifying the analysis. The modal properties of the equivalent reduced-DOF system, if known, can give a useful indication of how the passive crowd affects the modal properties of the occupied structure. Two equivalent reduced-DOF systems are investigated - SDOF and 3DOF systems. The errors between the responses of the equivalent systems and the full model are calculated and presented in the paper. The results show that the full model exhibits the behaviour of a SDOF system for structures with natural frequencies less than $4 \mathrm{~Hz}$ (when empty), whereas for structures with natural frequencies above $4 \mathrm{~Hz}$ the equivalent 3DOF system gives a better fit to the full model.
\end{abstract}

Keywords: Structural dynamics, human-structure interaction, crowd modelling

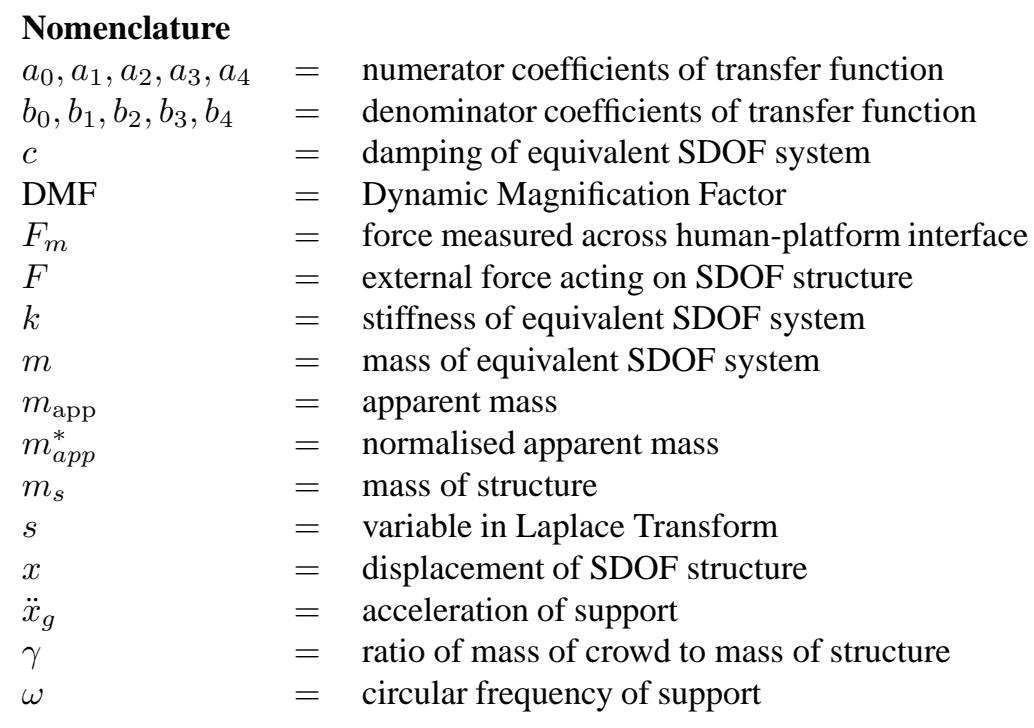

${ }^{*}$ Corresponding author. E-mail: Jackie.Sim@mottmac.com. 


\section{Introduction}

Excessive vibration is becoming a significant issue in the design of cantilever grandstands because of the trend towards building long and slender unsupported seating decks for a higher crowd capacity and an unobstructed sightline. In addition, cantilever grandstands are subjected to increasingly lively human-induced loadings, especially in pop concerts where the crowds synchronise their movements with the music played. The rhythmic movements of the crowd give rise to oscillating forces that might excite one of the low-frequency modes of long span cantilever tiers and cause resonant or near resonant behaviour. To date, vibration problems have been reported on large football stadiums in Brazil [1,2], Netherlands [3] and the UK [4,5] during pop concerts and football matches when the crowd was moving rhythmically.

In view of this possibility, the British Standard BS6399: Part 1 [6] warns specifically that a dynamic analysis should be performed on structures that are used for rhythmic activities and have natural frequencies below $8.4 \mathrm{~Hz}$ in the vertical direction when empty. A similar strategy is adopted by the Guide to Safety at Sports Grounds [7] but for structures with natural frequencies below $6 \mathrm{~Hz}$ when occupied by spectators. Interim guidance was issued by the IStructE/ODPM/DCMS working group [8] to address the immediate concern for public safety in sports grounds.

Several experimental studies and onsite measurements have been conducted to investigate the dynamic behaviour of cantilever grandstands. Experimental tests conducted on simple structures show contradictory findings [9,10]. Vibration tests on an $18.68 \mathrm{~Hz}$ beam [9] showed an increase in the natural frequency when the beam was occupied by a seated or standing person while another vibration test on a $4 \mathrm{~Hz}$ SDOF platform [10] showed a reduction in the natural frequency. Onsite modal tests of several grandstands [9,11-13] showed that the dynamic characteristics of the structures when occupied by a passive crowd (seated or standing) were different from when they were empty. Littler [11,12] conducted modal tests on three cantilever grandstands having natural frequencies ranging from 4 to $6 \mathrm{~Hz}$ when empty. Reynolds et al. [13] conducted modal tests on a stand at the Bradford Stadium during 20 football matches and 9 rugby matches. The stand had a series of closely spaced modes - the first six modes were between $3.28 \mathrm{~Hz}$ to $5.75 \mathrm{~Hz}$. Ellis and Ji [9] conducted modal tests on the Twickenham Stadium which had a natural frequency of $7.32 \mathrm{~Hz}$ when empty. All of these tests showed that the occupied structures had lower natural frequencies and higher damping than when they were empty. A higher mode was also observed in the Twickenham Stadium case. All these findings point to the need to model the passive crowd as a dynamic system added to the structural system.

Earlier work by the authors [14] modelled the seated and standing crowds as two independent two-degree-offreedom (2DOF) systems added to a single-degree-of-freedom (SDOF) structural system. Dynamic analyses were performed on the joint system for SDOF structures with empty natural frequencies varying from $1 \mathrm{Ho} 10 \mathrm{~Hz}$. The effect of the passive crowd was quantified in terms of the changes in peak Dynamic Magnification Factor (DMF) and resonant frequency. The DMF is defined as the peak dynamic displacement of the structure divided by its static deflection. This paper looks at representing the joint crowd-SDOF system as equivalent SDOF and 3DOF systems. The reduced-DOF systems will make the analysis more straightforward because fewer DOFs are used to model the joint crowd-SDOF system. In addition, the modal properties (mass, stiffness and damping) of the equivalent SDOF system will give some insight into how the passive crowd alters the dynamic response of the structure, e.g. by adding mass or damping, depending on the natural frequency of the structure.

This paper contains three sections. The first gives some relevant information on the full crowd-SDOF system. For a detailed description of the work on the full model, refer to [14]. The following two sections present the work on representing the full crowd-SDOF system as equivalent SDOF and 3DOF systems.

\section{The full crowd-structure model}

This section describes briefly the development of a 2DOF crowd model and the analysis of the full crowd-SDOF model, as reported in detail in [14]. 

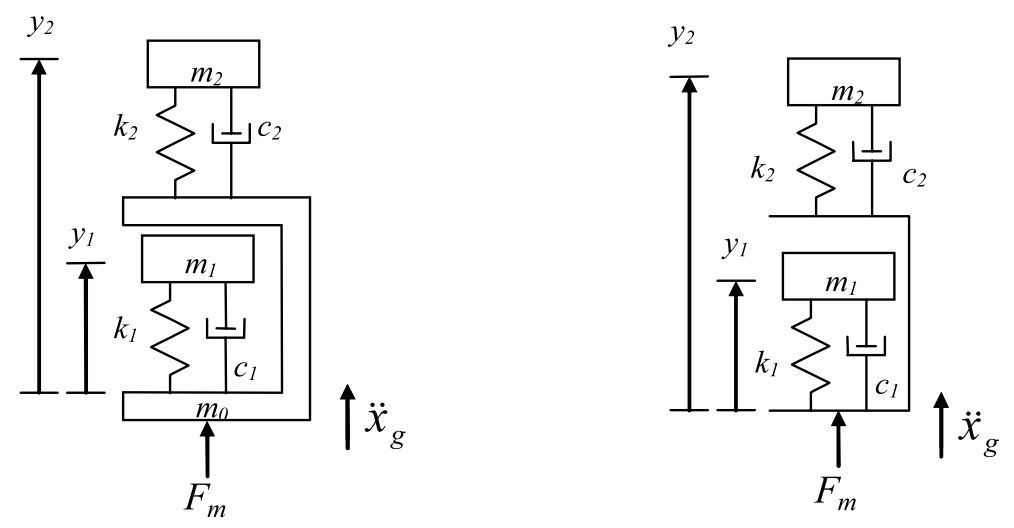

Fig. 1. Lumped parameter model for a seated human (left) and a standing human (right), reproduced from [16,18].

\subsection{Crowd model}

In [14], crowd models were derived based on the research work conducted on single seated and standing individuals by Griffin and co-workers [15-18]. In their experiments on 60 seated subjects ( 24 men, 24 women and 12 children) and on 12 standing subjects (all men), each subject was placed on a vibrating platform and shaken with a sine sweep at an acceleration level of $1.0 \mathrm{~m} / \mathrm{s}^{2}$ r.m.s. over the frequency range of 0 to $20 \mathrm{~Hz}$. The force across the human-structure interface and the acceleration of the platform were measured. Newton's Second Law states that the force acting on a mass is equal to the mass times its acceleration. Therefore, the force measured across the human-platform interface, $F_{m}$, divided by the acceleration of the platform, $\ddot{x}_{g}$, gives the 'dynamic mass' of the human body over a frequency range, referred to as the apparent mass of the human body:

$$
m_{\text {app }}(\omega)=\frac{F_{m}(\omega)}{\ddot{x}_{g}(\omega)}
$$

where $\omega$ is the circular frequency of the platform.

Griffin and co-workers fitted various lumped parameter models to the measured apparent mass-frequency response data of each test subject over the frequency range of 0 to $20 \mathrm{~Hz}$. They found that a $2 \mathrm{DOF}$ system gave the best fit to both seated and standing individuals, reproduced in schematic form in Fig. 1. The only difference in form between the two models is that the seated model has a rigid mass component in addition to the two moving masses.

The values of the model parameters $m_{0}, m_{1}, m_{2}, c_{1}, c_{2}, k_{1}$ and $k_{2}$ were published in [16 - Table 2] for 60 seated subjects and in [18 - Table 5] for 12 standing subjects. Using these published values, the apparent mass frequency responses of all models were calculated and summed to give the total response of each group of men, women and children. The total response was then normalised by the total mass of each group, giving the mean normalised apparent mass of each group. The mean normalised apparent mass over the frequency range of $0 \mathrm{to} 20 \mathrm{~Hz}$ was curve-fitted with rational polynomials of varying order from 1 to 6 using the damped Gauss-Newton method [19]. It was found that the solution converged with polynomials of order of 4 , i.e. there was no further significant reduction in the error when increasing the order from 4 to 5 . An order of 4 for the transfer function (given in Eq. (2) below) indicates that the group response can also be modelled as a 2DOF system. In other words, a crowd model consisting of numerous, different, parallel 2DOF systems, with each system representing one human, can be approximated well by using a single 2 DOF system.

$$
m_{\text {app }}^{*}(s)=\frac{a_{4} s^{4}+a_{3} s^{3}+a_{2} s^{2}+a_{1} s+a_{0}}{b_{4} s^{4}+b_{3} s^{3}+b_{2} s^{2}+b_{1} s+b_{0}}
$$

where $m_{\mathrm{app}}^{*}$ is the normalised apparent mass, $s$ is the Laplace Transform variable, and $a_{n}$ and $b_{n}$ are the coefficients defining the transfer function for $n$ varying from 0 to 4 .

The transfer function for a group of people is thus given by multiplying Eq. (2) by the total mass of the group.

Dynamic analyses were performed on a SDOF model of the structure occupied with a group of people, as shown in Fig. 2. The SDOF structure is assumed to have an intrinsic structural damping ratio of $2 \%$ and is subjected to 




Fig. 2. Joint crowd-SDOF system.

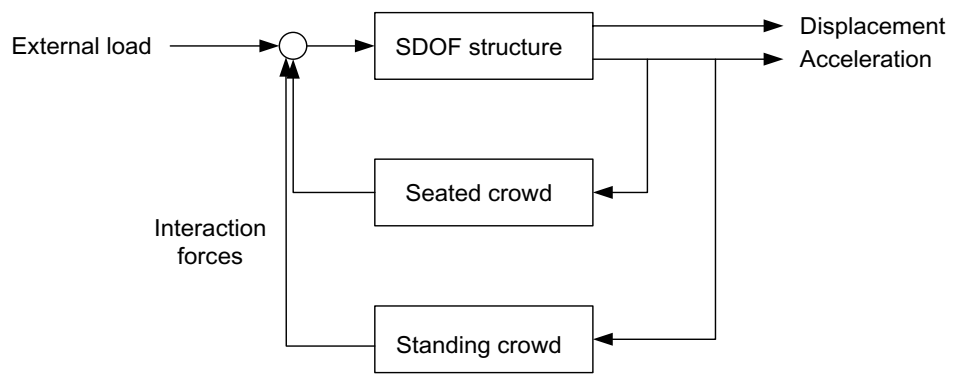

Fig. 3. Feedback system representation for the joint crowd-SDOF system.

an external sinusoidal force, $F$. It is assumed that $50 \%$ of the crowd are seated men and $50 \%$ are standing men. Dynamic analyses of the crowd-SDOF system were performed using the MATLAB ${ }^{\circledR}$ Control Toolbox [20] in which the crowd-SDOF system is represented as a feedback system, shown in Fig. 3. Each block represents one system: the SDOF structure system was defined by a state space model and each of the two seated and standing crowds by the transfer function in Eq. (2). Both seated and standing crowd systems are connected in feedback loops to the SDOF structure system. The interaction forces between the crowds and the structure are calculated from the acceleration feedback of the structure.

Analyses were carried out for natural frequencies of the empty structure varying from 1 to $10 \mathrm{~Hz}$ and mass ratios (actual mass of the passive crowd occupying the structure to mass of the empty structure) of $0,0.05,0.1,0.2,0.3$ and 0.4. The results were obtained in terms of the DMF. As an example, the DMF-frequency response curves for a $6 \mathrm{~Hz}$ structure are shown in Fig. 4 for various mass ratios.

For a structure with only a seated or a standing crowd, the joint crowd-structure system is a 3DOF system while for a structure with both standing and seated crowds, because each of the seated and standing crowds is modelled as an independent 2DOF system, the joint crowd-structure system is a 5DOF system. This paper looks at reducing a 50:50 seated/standing-SDOF system, which is a 5DOF system, to SDOF and 3DOF systems.

\section{Equivalent SDOF system}

\subsection{Curve-fitting DMF-frequency response curves}

In [14], the dynamic response of the full model shows that a passive crowd has a strong influence on the joint dynamical response by contributing added mass, damping and stiffness to the joint system. It was suggested by Michael Willford [21] that the joint system can be reduced to a SDOF system with modal properties different from 


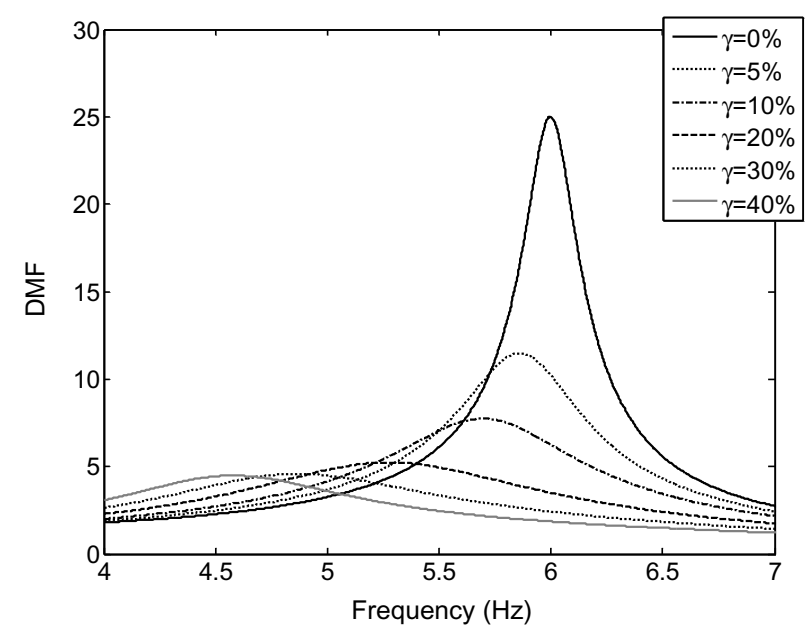

Fig. 4. DMF-frequency response curves for a $6 \mathrm{~Hz}$ structure with 50:50 seated/standing crowd.

those of the empty structure. In this paper, an equivalent SDOF system was fitted to the DMF-frequency response curves of the full model.

The equation of motion of a SDOF system subjected to a force of $F(t)$ is given by:

$$
m \ddot{x}(t)+c \dot{x}(t)+k x(t)=F(t)
$$

where $m, c$ and $k$ are the mass, damping and stiffness respectively.

Taking the Laplace Transform:

$$
m s^{2} X(s)+c s X(s)+k X(s)=F(s)
$$

Rearranging:

$$
X(s)=\frac{1}{m s^{2}+c s+k} \times F(s)
$$

If $X_{0}$ is the deflection under the load as if it were applied statically:

$$
X_{0}(s)=\frac{F(s)}{k}
$$

Normalising Eqn. 5 by $X_{0}$, gives the dynamic magnification factor (DMF):

$$
D M F(s)=\frac{X(s)}{X_{0}}=\frac{k}{m s^{2}+c s+k}
$$

For a DMF-frequency response curve, the most crucial response is around the peak response. Therefore, Eq. (7) was fitted over a bandwidth covering $1 / \sqrt{2}$ times the peak response using the damped Gauss-Newton method [19]. The modal properties of the equivalent SDOF system were obtained from the coefficients of the transfer function in Eq. (7). They were expressed as mass, stiffness and damping ratios. The mass and stiffness ratios are ratios of the mass and stiffness of the equivalent SDOF system to those of the empty structure. The damping ratio is that of the equivalent SDOF system, i.e. ratio of the damping to the critical value.

\subsection{Modal properties of equivalent SDOF system}

The results, presented in Fig. 5 for a SDOF structure with an intrinsic damping ratio of $2 \%$, show that for empty structures with low natural frequencies, the passive crowd adds both mass and stiffness to the system. For empty natural frequencies greater than about $5 \mathrm{~Hz}$, the passive crowd adds significant damping to the system. The damping ratio increases with increasing mass ratio, with the greatest damping ratio found for empty structures with natural frequencies between 5 and $9 \mathrm{~Hz}$. 



Fig. 5. Mass, stiffness and damping ratios for the equivalent SDOF system of a 50:50 seated/standing joint crowd-SDOF system, for a structure having a $2 \%$ damping ratio when empty.

\subsection{Error analysis}

For assessing the accuracy of the equivalent SDOF system in representing the full model, the peak DMF relative error and resonant frequency relative error were calculated. They are defined as (see also Fig. 6) the peak DMF relative error:

$$
\mathrm{DMF}_{\mathrm{RE}}^{\prime}=\frac{\Delta \mathrm{DMF}^{\prime}}{\mathrm{DMF}_{\text {Full model }}^{\prime}} \times 100 \%
$$




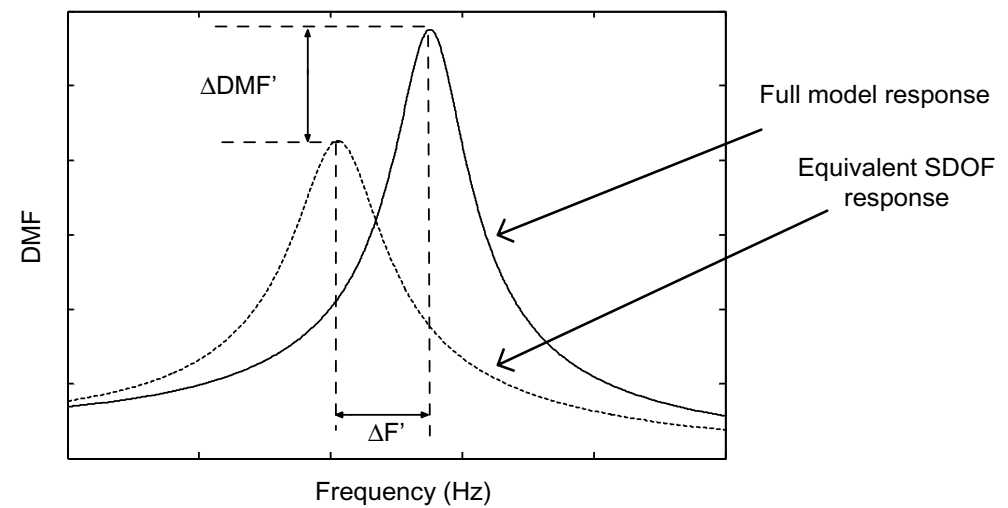

Fig. 6. Illustrations on peak DMF and resonant frequency relative errors.
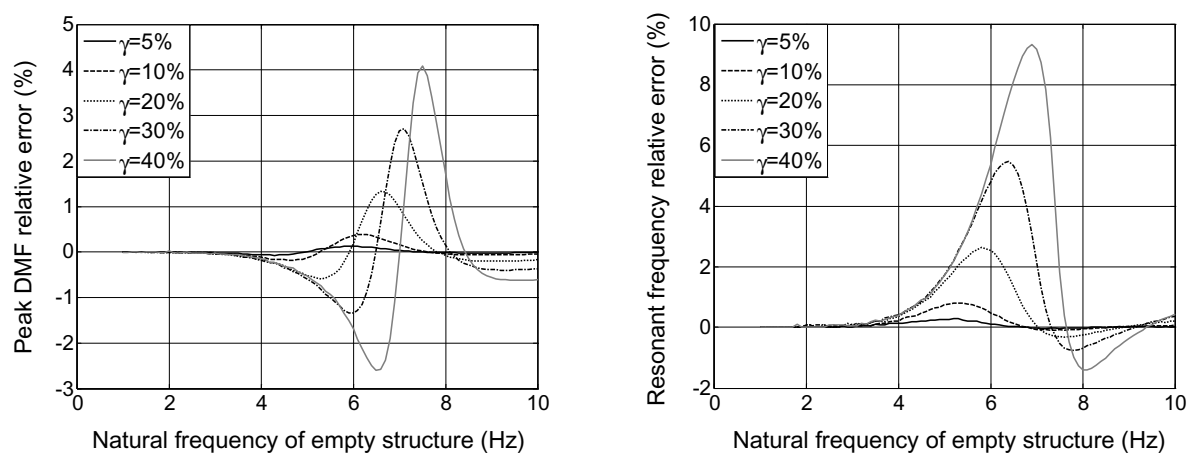

Fig. 7. Peak DMF relative error (left) and resonant frequency relative error (right) for equivalent SDOF system.

where DMF' Full model is the peak DMF of the full model, and $\triangle \mathrm{DMF}^{\prime}$ is the peak DMF of the equivalent SDOF minus the peak DMF of the full model, and the resonant frequency relative error:

$$
\mathrm{F}_{\mathrm{RE}}^{\prime} \frac{\Delta \mathrm{F}^{\prime}}{\mathrm{F}_{\text {Full model }}^{\prime}} \times 100 \%
$$

where the resonant frequency refers to the frequency at the peak DMF, F' Full model is the resonant frequency of full model and $\Delta \mathrm{F}^{\prime}$ the resonant frequency of the equivalent SDOF minus the resonant frequency of the full model.

Figure 7 shows that the equivalent SDOF system gives a very good fit for structures with natural frequencies up to $4 \mathrm{~Hz}$. This implies that the joint system exhibits the behaviour of a SDOF system, with the crowd adding mass to the SDOF structure. For structures with natural frequencies above $4 \mathrm{~Hz}$, the relative errors increase with increasing mass ratio, with maximum values of $4 \%$ for the peak DMF relative error and $9 \%$ for the resonant frequency relative error, for a mass ratio of 0.4. Therefore, with increasing number of people on the structure, the joint system behaves less like a SDOF system.

\section{Equivalent 3DOF system}

For the equivalent 3DOF system, each of the seated and standing crowd was modelled as a SDOF system, added to the SDOF structural system. The method of analysis is the same as the full model, i.e. represented as a feedback system as shown in Fig. 3 and analysed for its DMF-frequency response using MATLAB ${ }^{\circledR}$ Control Toolbox [20]. 
Table 1

Coefficients for transfer functions defining the crowd models for various groups (Eq. 10)

\begin{tabular}{lccrrrr}
\hline & $a_{2}$ & $a_{1}$ & \multicolumn{1}{c}{$a_{0}$} & $b_{2}$ & \multicolumn{1}{c}{$b_{1}$} & \multicolumn{1}{c}{$b_{0}$} \\
\hline Seated men & 0.2805 & 21.20 & 994.9 & 1 & 20.55 & 994.4 \\
Seated women & 0.2846 & 22.85 & 927.1 & 1 & 22.41 & 933.9 \\
Seated children & 0.1400 & 26.88 & 1096.0 & 1 & 27.15 & 1094.0 \\
Standing men & 0.2135 & 29.50 & 1454.0 & 1 & 29.39 & 1454.0 \\
\hline
\end{tabular}


Fig. 8. Mean normalised apparent mass of seated (left) and standing (right) men plotted with fitted SDOF and 2DOF models.

\subsection{SDOF crowd model}

For civil engineering structures, there are significant motions for structures with natural frequencies less than $8.4 \mathrm{~Hz}$ [6]. For this reason, the SDOF crowd model was obtained by curve-fitting a rational polynomial to the mean normalised apparent mass at low frequencies. Values of normalised apparent mass at frequencies above the curve-fitting range were obtained by extrapolation.

The transfer function for a SDOF crowd model is given by:

$$
m_{\text {app }}^{*}(s)=\frac{a_{2} s^{2}+a_{1} s+a_{0}}{b_{2} s^{2}+b_{1} s+b_{0}}
$$

Equation (10) was fitted over various frequency bandwidths, such as 0 to $5 \mathrm{~Hz}, 0$ to $8 \mathrm{~Hz}$ and 0 to $10 \mathrm{~Hz}$. By trial and error, it was found that curve-fitting from 0 to $5 \mathrm{~Hz}$ for the mean normalised apparent mass gave the least total absolute error over the range 0 to $8 \mathrm{~Hz}$.

Figure 8 shows the mean normalised apparent mass for groups of seated and standing men, together with the fitted transfer functions given in Eq. (2) for a 2DOF crowd model and in Eq. (10) for a SDOF crowd model. While the 2DOF crowd model shows an almost perfect fit, the SDOF crowd model shows deviation at high frequencies. The values of the coefficients in Eq. (10) are presented in Table 1.

\subsection{Dynamic analysis and error analysis}

Dynamic analyses similar to the full model were performed for the equivalent 3DOF model. The peak DMF relative error and resonant frequency relative error are presented in Fig. 9. The relative errors increase proportionally with the mass ratio because the crowd model is obtained by multiplying the transfer function in Eq. (10) by the mass of the passive crowd. For the peak DMF relative error, it falls within the range $\pm 3 \%$. The resonant frequency relative error is very low, less than $3 \%$. 

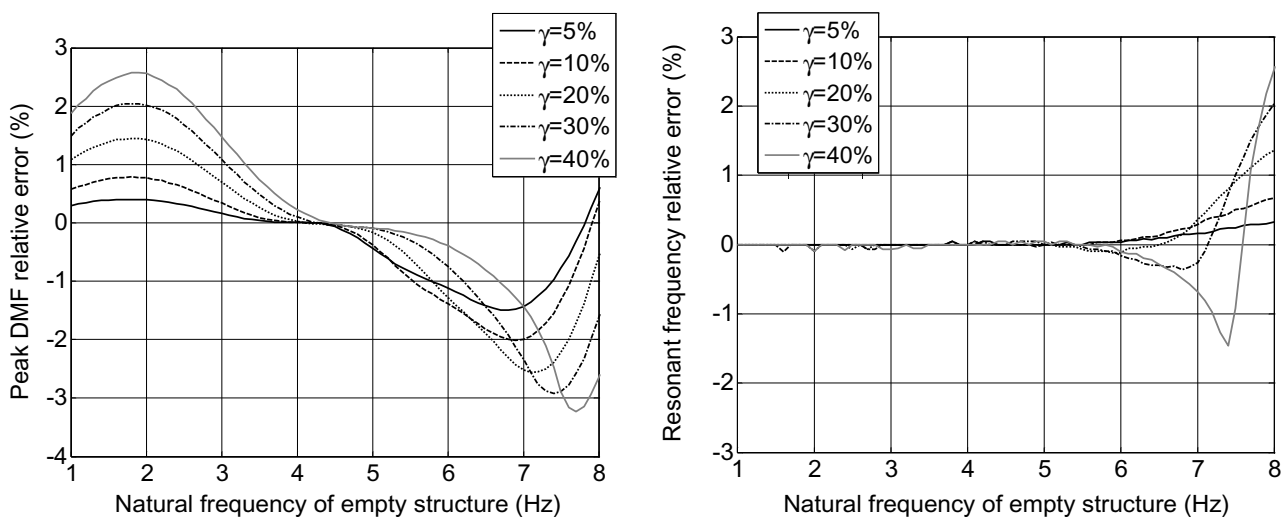

Fig. 9. Peak DMF relative error (left) and resonant frequency relative error (right) for equivalent 3DOF system.
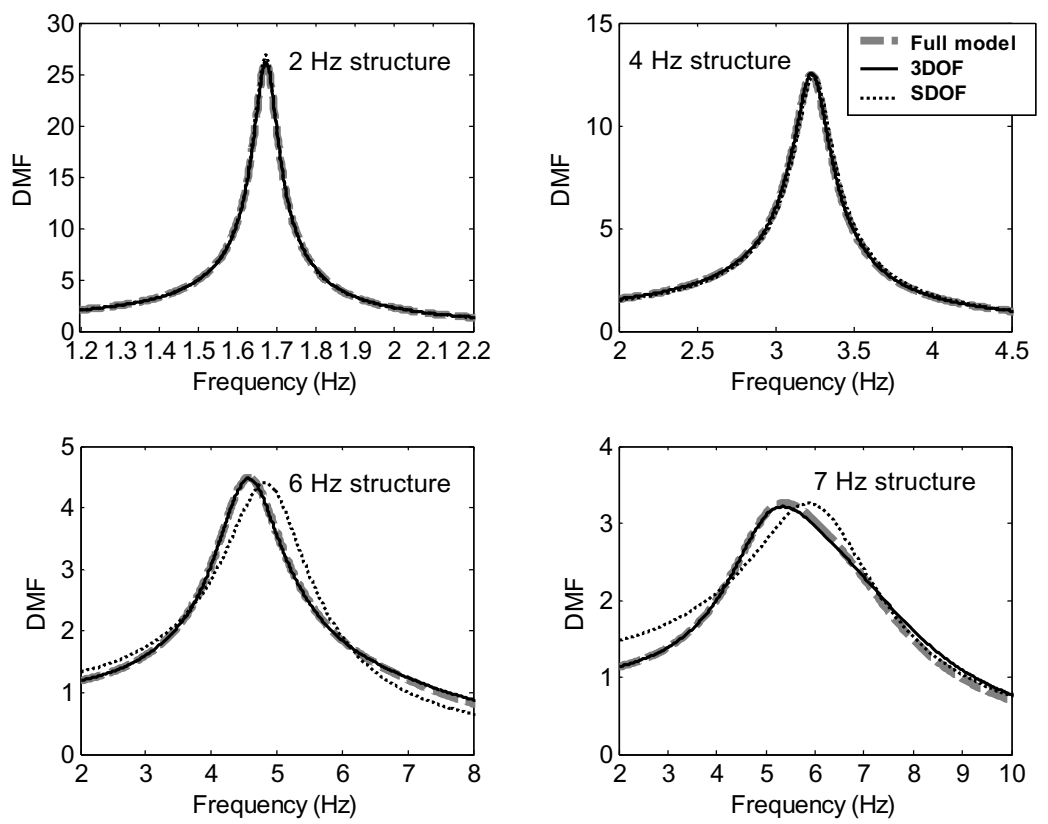

Fig. 10. DMF-frequency response curves for 2, 4, 6 adnd $7 \mathrm{~Hz}$ structures with mass ratio of 0.4 . Each plot shows the responses of full model, equivalent SDOF and 3DOF models. The natural frequency refers to that of the structure when it is empty.

\subsection{Bode diagrams}

Figure 10 shows the DMF-frequency response curves for the full model, equivalent SDOF and 3DOF systems, for structures with natural frequencies of 2, 4, 6 and $7 \mathrm{~Hz}$ (when empty) and a mass ratio of 0.4. The equivalent SDOF shows a good fit for the 2 and $4 \mathrm{~Hz}$ structures. However, for the 6 and $7 \mathrm{~Hz}$ structures, the shape of the curve does not match that of the full model. In particular, there is a discrepancy in the resonant frequency but a good approximation of the maximum DMF is found. As for the equivalent 3DOF system, the shape of the curve matches and it gives good approximations for both the resonant frequency and the peak DMF.

\section{Conclusions}

The results show that the joint crowd-SDOF system can be modelled satisfactorily as a SDOF system for structures with empty natural frequencies of 1 to $4 \mathrm{~Hz}$. At low natural frequencies of the empty structure, the crowd adds 
mass to the structure and thus the joint system exhibits little deviation from the behaviour of a SDOF system. For structures above $4 \mathrm{~Hz}$, the relative errors in DMF and resonant frequency increase with the mass ratio, indicating a deviation from the behaviour of a SDOF system as the mass of the people on the structure increases. Bode plots of the equivalent 3DOF system show a good match with those of the full model while a mismatch is found for the equivalent SDOF system for high natural frequency structures. Hence the equivalent 3DOF system is found to give a more accurate fit for structures above $4 \mathrm{~Hz}$ due to a smaller error in the resonant frequency.

In practice, the engineer is interested in the maximum DMF. The slight discrepancy in the resonant frequency given by the equivalent SDOF is of secondary importance. The maximum relative errors in DMF for the equivalent SDOF and 3DOF systems are both within the range of $\pm 4 \%$. In fact, the equivalent SDOF system gives a better fit for structures below $4 \mathrm{~Hz}$. Therefore, for design purpose, the equivalent SDOF, with fewer DOFs, is a better option because it is simpler. It should be noted that theoretically, the equivalent 3DOF is a more accurate model because it gives a lower resonant frequency relative error.

\section{Acknowledgements}

The authors would like to acknowledge valuable inputs from members of the IStructE/ODPM/DCMS working group. In particular to Mike Willford for having the original idea.

\section{References}

[1] R.C. Batista and C. Magluta, Spectator-induced vibration of Maracana Stadium, EURODYN '93 Trondheim, Norway, 21-23 June 1993, 985-992.

[2] P.A.O. Almeida and J.F.S. Rodrigues, Investigation of the vibrations induced by people in soccer stadiums, 1998 SEM Spring Conference and Exhibition, Houston, Texas, 1998, 271-274.

[3] P.V. Staalduinen and W. Courage, Dynamic loading of Feyenoord stadium during pop concerts, Symposium: Places of assembly and long-span building structures, Birmingham, UK, 7-9 September 1994, Report 71, International Association for Bridge and Structural Engineering (IABSE), 1994, 283-288.

[4] D. Rogers, Two more 'wobbly' stands, Construction News (17 August 2000).

[5] D. Rogers and R. Thompson, Liverpool stand gets a red card, Construction News (10 August 2000).

[6] British Standards Institution (BSI), BS 6399: Part 1: Code of practice for dead and imposed loads. British Standards Institution, London, 1996.

[7] Department of National Heritage and Scottish Office, Guide to Safety at Sports Grounds, 4th edition, Stationary Office, London. ISBN 0 $113000952,1997$.

[8] IStructE/ODPM/DCMS working group, Dynamic performance requirements for permanent grandstands subject to crowd actions. Interim guidance on assessment and design. London, UK, 2001.

[9] B.R. Ellis and T. Ji, Human-structure interaction in vertical vibrations, Structures and Buildings, the Proceedings of Institution of Civil Engineers 122(1) (1997), 1-9.

[10] R.E. Harrison and J.R. Wright, Combined active and passive human loading on a flexible platform, 23rd International Modal Analysis Conference (IMAC XXIII), Orlando, Florida, USA, 31 January-3 February 2005.

[11] J.D. Littler, Full-scale testing of large cantilever grandstands to determine their dynamic response, Stadia, arenas and grandstands, Thompson, P.D., Tollocako, J.J.A., J.N. Clarke, eds, E and FN Spon, London, 1998, pp. 123-134.

[12] J.D. Littler, The dynamic response of a three tier cantilever grandstand, in: Structural Dynamics - EURODYN '99, 1999, pp. 623-628.

[13] P. Reynolds, A. Pavic and Z. Ibrahim, Changes of modal properties of a stadium structure occupied by a crowd, 22nd International Modal Analysis Conference (IMAC XXII), Dearborn, Detroit, USA, 26-29 January 2004.

[14] J. Sim, A. Blakeborough and M.S. Williams, Modelling effects of passive crowds on grandstand vibration, Structures and Buildings, Proceedings of the Institution of Civil Engineers 159 (2006), 247-259.

[15] T.E. Fairley and M.J. Griffin, The apparent mass of the seated human body: vertical vibration, Journal of Biomechanics 22(2) (1989), 81-94.

[16] L. Wei and M.J. Griffin, Mathematical models for the apparent mass of the seated human body exposed to vertical vibration, Journal of Sound and Vibration 212(5) (1998), 855-874.

[17] Y. Matsumoto and M.J. Griffin, Dynamic response of the standing human body exposed to vertical vibration: influence of posture and vibration magnitude, Journal of Sound and Vibration 212(1) (1998), 85-107.

[18] Y. Matsumoto and M.J. Griffin, Mathematical models for the apparent masses of standing subjects exposed to vertical whole-body vibration, Journal of Sound and Vibration 260(3) (2003), 431-451.

[19] J.E. Dennis, Jr. and R.B. Schnabel, Numerical Methods for Unconstrained Optimization and Nonlinear Equations. Englewood Cliffs, NJ: Prentice-Hall, 1983.

[20] The MathWorks, Inc. MATLAB ${ }^{\circledR}$ Control Toolbox.

[21] A. Blakeborough, Personal communication (2005). 

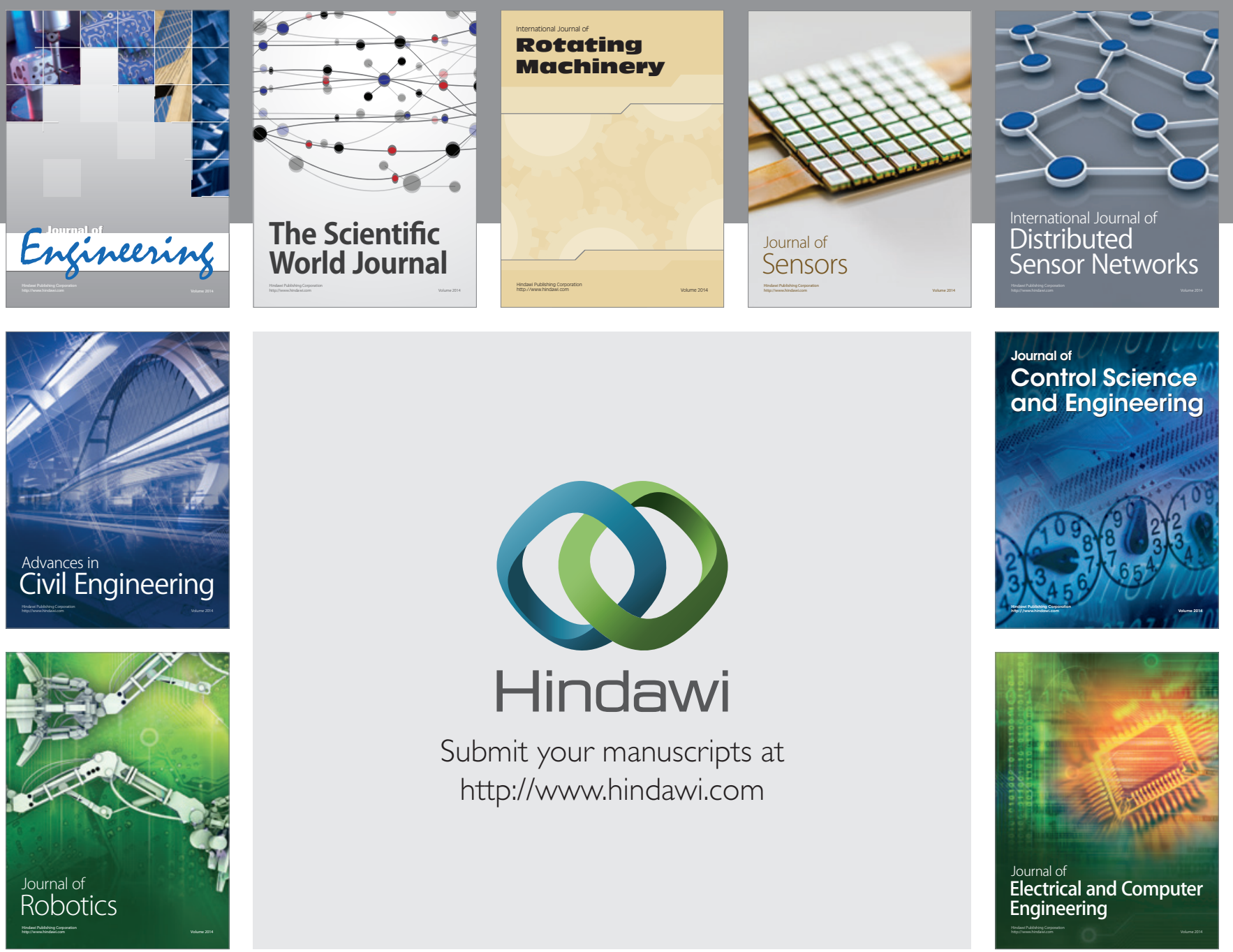

Submit your manuscripts at

http://www.hindawi.com
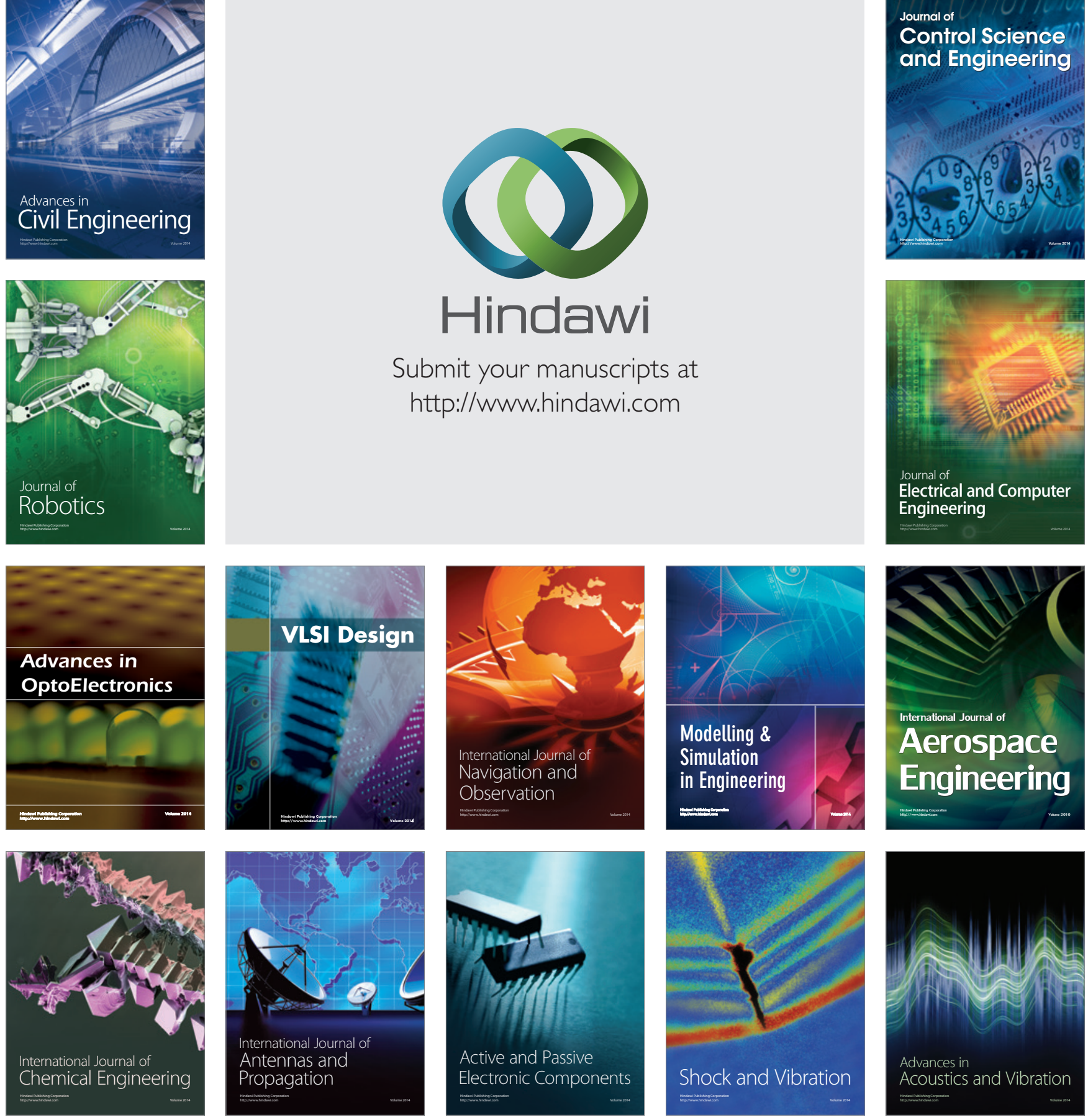